\title{
DIMENSIONS OF PRYM VARIETIES
}

\author{
AMY E. KSIR \\ (Received 22 January 2001)
}

\begin{abstract}
Given a tame Galois branched cover of curves $\pi: X \rightarrow Y$ with any finite Galois group $G$ whose representations are rational, we compute the dimension of the (generalized) Prym variety $\operatorname{Prym}_{\rho}(X)$ corresponding to any irreducible representation $\rho$ of $G$. This formula can be applied to the study of algebraic integrable systems using Lax pairs, in particular systems associated with Seiberg-Witten theory. However, the formula is much more general and its computation and proof are entirely algebraic.
\end{abstract}

2000 Mathematics Subject Classification. 14H40, 14H70, 81T13.

1. Introduction. The most familiar Prym variety arises from a (possibly branched) double cover $\pi: X \rightarrow Y$ of curves. In this situation, there is a surjective norm map $\mathrm{Nm}: \operatorname{Jac}(X) \rightarrow \operatorname{Jac}(Y)$, and the Prym (another Abelian variety) is a connected component of its kernel. Another way to think of this is that the involution $\sigma$ of the double cover induces an action of $\mathbb{Z} / 2 \mathbb{Z}$ on the vector space $H^{0}\left(X, \omega_{X}\right)$, which can then be decomposed as a representation of $\mathbb{Z} / 2 \mathbb{Z}$. The Jacobian of the base curve $Y$ and the Prym correspond to the trivial and sign representations, respectively. The Prym variety can be defined as the component containing the identity of $\left(\operatorname{Jac}(X) \otimes_{\mathbb{Z}} \varepsilon\right)^{\sigma}$, where $\varepsilon$ denotes the sign representation of $\mathbb{Z} / 2 \mathbb{Z}$.

The generalization of this construction that we study in this paper is as follows. Let $G$ be a finite group, and $\pi: X \rightarrow Y$ be a tame Galois branched cover, with Galois group $G$, of smooth projective curves over an algebraically closed field. The action of $G$ on $X$ induces an action on the vector space of differentials $H^{0}\left(X, \omega_{X}\right)$, and on the $\operatorname{Jacobian} \operatorname{Jac}(X)$. For any representation $\rho$ of $G$, we define $\operatorname{Prym}_{\rho}(X)$ to be the connected component containing the identity of $\left(\operatorname{Jac}(X) \otimes_{\mathbb{Z}} \rho^{*}\right)^{G}$. The vector space $H^{0}\left(X, \omega_{X}\right)$ decomposes as a $\mathbb{Z}[G]$-module into a direct sum of isotypic pieces

$$
H^{0}\left(X, \omega_{X}\right)=\bigoplus_{j=1}^{N} \rho_{j} \otimes V_{j},
$$

where $\rho_{1}, \ldots, \rho_{N}$ are the irreducible representations of $G$. If $G$ is such that all of its representations are rational, then the Jacobian also decomposes, up to isogeny, into a direct sum of Pryms [5]:

$$
\operatorname{Jac}(X) \sim \bigoplus_{j=1}^{N} \rho_{j} \otimes \operatorname{Prym}_{\rho_{j}}(X) .
$$

In particular, if $G$ is the Weyl group of a semisimple Lie algebra, then it satisfies this property. 
The goal of this paper is to compute the dimension of such a Prym variety. This formula is given in Section 2, with a proof that uses only the Riemann-Hurwitz theorem and some character theory. Special cases of this formula relevant to integrable systems have appeared previously [2, 11, 12, 13].

One motivation for this work comes from the study of algebraically integrable systems. An algebraically integrable system is a Hamiltonian system of ordinary differential equations, where the phase space is an algebraic variety with an algebraic (holomorphic, over $\mathbb{C}$ ) symplectic structure. The complete integrability of the system means that there are $n$ commuting Hamiltonian functions on the $2 n$-dimensional phase space. For an algebraically integrable system, these functions should be algebraic, in which case they define a morphism to an $n$-dimensional space of states for the system. The flow of the system will be linearized on the fibers of this morphism, which, if they are compact, will be $n$-dimensional Abelian varieties.

Many such systems can be solved by expressing the system as a Lax pair depending on a parameter $z$. The equations can be written in the form $(d / d t) A=[A, B]$, where $A$ and $B$ are elements of a Lie algebra $\mathfrak{g}$, and depend both on time $t$ and on a parameter $z$, which is thought of as a coordinate on a curve $Y$. In this case, the flow of the system is linearized on a subtorus of the Jacobian of a Galois cover of $Y$. If it can be shown that this subtorus is isogenous to a Prym of the correct dimension, then the system is completely integrable.

In Section 3, we briefly discuss two examples of such systems, the periodic Toda lattice and Hitchin systems. Both of these are important in Seiberg-Witten theory, providing solutions to $\mathcal{N}=2$ supersymmetric Yang-Mills gauge theory in four dimensions.

2. Dimensions. We can start by using the Riemann-Hurwitz formula to find the genus $g_{X}$ of $X$, which will be the dimension of the whole space $H^{0}\left(X, \omega_{X}\right)$ and of $\operatorname{Jac}(X)$. Since $\pi: X \rightarrow Y$ is a cover of degree $|G|$, we get

$$
g_{X}=1+|G|(g-1)+\frac{\operatorname{deg} R}{2},
$$

where $g$ is the genus of the base curve $Y$ and $R$ is the ramification divisor.

The first isotypic piece whose dimension we can find is $V_{1}$, corresponding to the trivial representation. The subspace where $G$ acts trivially is the subspace of differentials which are pullbacks by $\pi$ of differentials on $Y$. This tells us that $\operatorname{dim} V_{1}=$ $\operatorname{dim} H^{0}\left(Y, \omega_{Y}\right)=g$.

In the case of classical Pryms, where $G=\mathbb{Z} / 2$, there is only one other isotypic piece, $V_{\varepsilon}$ corresponding to the sign representation $\varepsilon$. Thus we have

$$
\operatorname{dim} V_{\varepsilon}=g_{X}-g=g-1+\frac{\operatorname{deg} R}{2} .
$$

For larger groups $G$, there are more isotypic pieces, but we also have more information: we can look at intermediate curves, that is, quotients of $X$ by subgroups $H$ of $G$. Differentials on $X / H$ pull back to differentials on $X$, where $H$ acts trivially. Thus

$$
H^{0}\left(X / H, \omega_{X / H}\right)=\bigoplus_{j=1}^{N}\left(\rho_{j}\right)^{H} \otimes V_{j} .
$$


The map $\pi_{H}: X / H \rightarrow Y$ will be a cover of degree $|G| /|H|$, so Riemann-Hurwitz gives us the following formula for the genus $g_{H}$ of $X / H$, which is the dimension of $H^{0}\left(X / H, \omega_{X / H}\right)$ :

$$
g_{H}=1+\frac{|G|}{|H|}(g-1)+\frac{\operatorname{deg} R_{H}}{2},
$$

where again $R_{H}$ is the ramification divisor.

We can further analyze the ramification divisor, by classifying the branch points according to their inertial groups. Since $\pi: X \rightarrow Y$ is a Galois cover of curves over $\mathbb{C}$, all of the inertial groups must be cyclic.

LEMMA 2.1. Let $G$ be a finite group all of whose characters are defined over $\mathbb{Q}$. If two elements $x, y \in G$ generate conjugate cyclic subgroups, then they are conjugate.

Proof (adapted from [3]). We want to show that for any character $\chi$ of $G, \chi(x)=$ $\chi(y)$. Then the properties of characters will tell us that $x$ and $y$ must be in the same conjugacy class.

We may assume that $x$ and $y$ generate the same subgroup $H$. Then $y=x^{k}$ for some integer $k$ relatively prime to $|H|$. Let $\chi$ be a character of $G$, and $\rho: G \rightarrow \operatorname{GL}(n, \mathbb{C})$ a representation with character $\chi$. Then $\rho(x)$ will be a matrix with eigenvalues $\lambda_{1}, \ldots, \lambda_{n}$, and $\rho(y)$ will have eigenvalues $\lambda_{1}^{k}, \ldots, \lambda_{n}^{k}$. Since $x^{|H|}=1$, we have $\lambda_{1}^{|H|}=\cdots=\lambda_{n}^{|H|}=1$. Let $\xi$ be a primitive $|H|$ th root of unity. Then we can write $\lambda_{1}=\xi^{\nu_{1}}, \ldots, \lambda_{n}=\xi^{\nu_{n}}$ for some integers $v_{i}$. Now $\chi(x)=\operatorname{Trace}(\rho(x))=\lambda_{1}+\cdots+\lambda_{n}$, and $\chi(y)=\chi\left(x^{k}\right)=$ $\lambda_{1}^{k}+\cdots+\lambda_{n}^{k}$. Thus $\chi(y)$ will be the image of $\chi(x)$ under the element of $\operatorname{Gal}(\mathbb{Q}(\xi) / \mathbb{Q})$ which sends $\xi \mapsto \xi^{k}$. Since the values of $\chi$ are rational, this element will act trivially, so $\chi(y)=\chi(x)$.

From now on, we suppose that $G$ is such that all of its characters are rational. (This is true, for instance, if $G$ is a Weyl group.) Pick representative elements $h_{1}, \ldots, h_{N}$ for each conjugacy class in $G$, and let $H_{1}, \ldots, H_{N}$ be the cyclic groups that each of them generates. By Lemma 2.1, this is the whole set (up to conjugacy) of cyclic subgroups of $G$. We can partially order this set of cyclic subgroups by their size, so that $H_{1}$ is the trivial subgroup. Now we can classify the branch points: let $R_{k}, k=2, \ldots, N$ be the degree of the branch locus with inertial group conjugate to $H_{k}$ (ignoring the trivial group). Over each point of the branch locus, where the inertial group is conjugate to $H_{k}$, there are $|G| /\left|H_{k}\right|$ points in the fiber. Thus the degree of the ramification divisor $R$ of $\pi: X \rightarrow Y$ is

$$
\operatorname{deg} R=\sum_{k=1}^{N}\left(|G|-\frac{|G|}{\left|H_{k}\right|}\right) R_{k}
$$

For each quotient curve $X / H$, each point in the fiber of $\pi_{H}: X / H \rightarrow Y$ over a point with inertial group $H_{k}$ corresponds to a double coset $H_{k} \backslash G / H$. Thus the degree of the ramification divisor $R_{H}$ is

$$
\operatorname{deg} R_{H}=\sum_{k=1}^{N}\left(\frac{|G|}{|H|}-\#\left(H_{k} \backslash G / H\right)\right) R_{k}
$$


Combining (2.5) and (2.6) with the earlier Riemann-Hurwitz computations ((2.1) and (2.4)), we get

$$
\begin{aligned}
& g_{X}=1+|G|(g-1)+\sum_{k}\left(|G|-\frac{|G|}{|H|}\right) \frac{R_{k}}{2} \\
& g_{H}=1+\frac{|G|}{|H|}(g-1)+\sum_{k}\left(\frac{|G|}{|H|}-\#\left(H_{k} \backslash G / H\right)\right) \frac{R_{k}}{2} .
\end{aligned}
$$

Since the genera $g_{H}$ are exactly the dimensions $\operatorname{dim} H^{0}\left(X / H, \omega_{X / H}\right)$, we also have

$$
g_{H}=\sum_{j=1}^{N} \operatorname{dim} \rho_{j}^{H} \operatorname{dim} V_{j} .
$$

For each subgroup $H$, this is a linear equation for the unknown $\operatorname{dimensions} \operatorname{dim} V_{j}$ in terms of the genus $g_{H}$. Thus by taking quotients by the set of all cyclic subgroups $H_{1} \cdots H_{N}$, we get a system of $N$ equations. We wish to invert the matrix $\operatorname{dim} \rho_{j}^{H_{i}}$ and find the $N$ unknowns $\operatorname{dim} V_{j}$.

LEMMA 2.2. The matrix $\operatorname{dim} \rho_{j}^{H_{i}}$ is invertible.

Proof. We show that the rows of the matrix are linearly independent, using the fact that rows of the character table are linearly independent. First, note that $\operatorname{dim} \rho_{j}^{H_{i}}$, the dimension of the subspace of $\rho_{j}$ invariant under $H_{i}$, is equal to the inner product of characters $\left\langle\operatorname{Res}_{H_{i}}^{G} \rho_{j}, \mathbf{1}\right\rangle$, which we can read off from the character table of $G$ as

$$
\operatorname{dim} \rho_{j}^{H_{i}}=\frac{1}{\left|H_{i}\right|} \sum_{a_{i} \in H_{i}} \chi_{\rho_{j}}\left(a_{i}\right) .
$$

Compare this matrix to the matrix of the character table $\chi_{\rho_{j}}\left(a_{i}\right)$. From (2.9) we see that each row is a sum of multiples of rows of the character table. Since each element of a subgroup has order less than or equal to the order of the subgroup, the rows of the character table being added to get row $i$ appear at or below row $i$ in the character table. Thus if we write the matrix $\operatorname{dim} \rho_{j}^{H_{i}}$ in terms of the basis of the character table, we get a lower triangular matrix with nonzero entries on the diagonal. By row reduction, we see that the linear independence of the rows of $\operatorname{dim} \rho_{j}^{H_{i}}$ is equivalent to the linear independence of the rows of the character table.

THEOREM 2.3. For each nontrivial irreducible representation $\rho_{j}$ of $G, V_{j}$ has dimension

$$
\left(\operatorname{dim} \rho_{j}\right)(g-1)+\sum_{k=1}^{N}\left(\left(\operatorname{dim} \rho_{j}\right)-\left(\operatorname{dim} \rho_{j}^{H_{k}}\right)\right) \frac{R_{H_{k}}}{2} .
$$

Proof. Since the matrix $\operatorname{dim} \rho_{j}^{H_{i}}$ is invertible, there is a unique solution to the system of (2.8), so we only need to show that this is a solution. Namely, given this formula for $\operatorname{dim} V_{j}$ and combining (2.7) and (2.8), we wish to show that for each cyclic subgroup $H_{i}$,

$$
\sum_{j=1}^{N} \operatorname{dim} \rho_{j}^{H_{i}} \operatorname{dim} V_{j}=1+\frac{|G|}{\left|H_{i}\right|}(g-1)+\sum_{k}\left(\frac{|G|}{\left|H_{i}\right|}-\#\left(H_{k} \backslash G / H_{i}\right)\right) \frac{R_{k}}{2} .
$$


Note that on the left-hand side we are summing over all representations, not just the nontrivial ones, so our notation will be simpler if we write $\operatorname{dim} V_{1}=g$ in a similar form to (2.8). For the trivial representation $\rho_{1},\left(\operatorname{dim} \rho_{1}\right)-\left(\operatorname{dim} \rho_{1}^{H_{k}}\right)=0$ (since $\rho_{1}$ is fixed by any subgroup $H_{k}$ ), so

$$
\operatorname{dim} V_{1}=1+\left(\operatorname{dim} \rho_{1}\right)(g-1)+\sum_{k=1}^{N}\left(\left(\operatorname{dim} \rho_{1}\right)-\left(\operatorname{dim} \rho_{1}^{H_{k}}\right)\right) \frac{R_{H_{k}}}{2}
$$

The sum on the left-hand side of (2.11) will be

$$
1+\sum_{j=1}^{N} \operatorname{dim} \rho_{j}^{H_{i}}\left(\left(\operatorname{dim} \rho_{j}\right)(g-1)+\sum_{k=1}^{N}\left(\left(\operatorname{dim} \rho_{j}\right)-\left(\operatorname{dim} \rho_{j}^{H_{k}}\right)\right) \frac{R_{H_{k}}}{2}\right) .
$$

We look at the $(g-1)$ term and the $R_{H_{k}}$ terms separately. For the $(g-1)$ coefficient, we can write both $\operatorname{dim} \rho_{j}^{H_{i}}$, and $\operatorname{dim} \rho_{j}$ in terms of characters of $G$ (as in (2.9)) and exchange the order of summation to get

$$
\sum_{j=1}^{N} \operatorname{dim} \rho_{j}^{H_{i}} \operatorname{dim} \rho_{j}=\frac{1}{\left|H_{i}\right|} \sum_{a_{i} \in H_{i}} \sum_{j=1}^{N} \chi_{\rho_{j}}\left(a_{i}\right) \chi_{\rho_{j}}(e),
$$

where $e$ is the identity element of $G$. The inner sum amounts to take the inner product of two columns of the character table of $G$. The orthogonality of characters tells us that this inner product will be zero unless the two columns are the same, in this case if $a_{i}=e$. Thus the sum over elements in $H_{i}$ disappears, and we get the sum of the squares of the dimensions of the characters

$$
\frac{1}{\left|H_{i}\right|} \sum_{j=1}^{N} x_{\rho_{j}}(e)^{2}=\frac{|G|}{\left|H_{i}\right|}
$$

which is what we want.

The $R_{H_{k}}$ term looks like

$$
\sum_{j=1}^{N} \operatorname{dim} \rho_{j}^{H_{i}} \sum_{k=1}^{N}\left(\left(\operatorname{dim} \rho_{j}\right)-\left(\operatorname{dim} \rho_{j}^{H_{k}}\right)\right) \frac{R_{H_{k}}}{2} .
$$

We can distribute and rearrange the sums to get

$$
\sum_{k=1}^{N}\left(\sum_{j=1}^{N} \operatorname{dim} \rho_{j}^{H_{i}} \operatorname{dim} \rho_{j}-\sum_{j=1}^{N} \operatorname{dim} \rho_{j}^{H_{i}} \operatorname{dim} \rho_{j}^{H_{k}}\right) \frac{R_{H_{k}}}{2} .
$$

As in (2.14) and (2.15), the first term becomes $|G| /\left|H_{i}\right|$. The second term is also the inner product of columns of the character table:

$$
\sum_{j=1}^{N} \operatorname{dim} \rho_{j}^{H_{i}} \operatorname{dim} \rho_{j}^{H_{k}}=\frac{1}{\left|H_{i}\right|} \frac{1}{\left|H_{k}\right|} \sum_{a_{i} \in H_{i}} \sum_{a_{k} \in H_{k}} \sum_{j=1}^{N} \chi_{\rho_{j}}\left(a_{i}\right) \chi_{\rho_{j}}\left(a_{k}\right) .
$$


This will be zero unless $a_{i}$ and $a_{k}$ are conjugate, in which case $\chi_{\rho_{j}}\left(a_{i}\right)=\chi_{\rho_{j}}\left(a_{k}\right)$ and character theory tells us (cf. [8, page 18]) that

$$
\sum_{j=1}^{N} \chi_{\rho_{j}}\left(a_{i}\right)^{2}=\frac{|G|}{c\left(a_{i}\right)}
$$

where $c\left(a_{i}\right)$ is the number of elements in the conjugacy class of $a_{i}$. Now the second term has become

$$
\frac{|G|}{\left|H_{i}\right|\left|H_{k}\right|} \sum_{\left\{a_{i}, a_{k}\right\}} \frac{1}{c\left(a_{i}\right)},
$$

where the sum is taken over pairs of elements $a_{i} \in H_{i}, a_{k} \in H_{k}$ such that $a_{i}$ and $a_{k}$ are conjugate. This is exactly the number of double cosets $\#\left(H_{k} \backslash G / H_{i}\right)$.

Adding up all of the terms, the sum on the left-hand side becomes

$$
1+\frac{|G|}{\left|H_{i}\right|}(g-1)+\left(\frac{|G|}{\left|H_{i}\right|}-\#\left(H_{k} \backslash G / H_{i}\right)\right) \frac{R_{H_{k}}}{2},
$$

which is exactly the right-hand side.

COROLlaRY 2.4. For each nontrivial irreducible representation $\rho_{j}$ of $G, \operatorname{Prym}_{\rho_{j}}(X)$ has dimension

$$
\left(\operatorname{dim} \rho_{j}\right)(g-1)+\sum_{k=1}^{N}\left(\left(\operatorname{dim} \rho_{j}\right)-\left(\operatorname{dim} \rho_{j}^{H_{k}}\right)\right) \frac{R_{H_{k}}}{2} .
$$

\section{Integrable systems}

3.1. Periodic Toda lattice. The periodic Toda system is a Hamiltonian system of differential equations with Hamiltonian

$$
H(p, q)=\frac{|p|^{2}}{2}+\sum_{\alpha} e^{\alpha(q)},
$$

where $p$ and $q$ are elements of the Cartan subalgebra $t$ of a semisimple Lie algebra $\mathfrak{g}$, and the sum is over the simple roots of $\mathfrak{g}$ plus the highest root. This system can be expressed in Lax form [1] $(d / d t) A=[A, B]$, where $A$ and $B$ are elements of the loop algebra $\mathfrak{g}^{(1)}$, and can be thought of as elements of $\mathfrak{g}$ which depend on a parameter $z \in \mathbb{P}^{1}$. For $\mathfrak{s}^{[}(n), A$ is of the form

$$
\left(\begin{array}{cccc}
y_{1} & 1 & & x_{0} z \\
x_{1} & y_{2} & \ddots & \\
& \ddots & \ddots & 1 \\
z & & x_{n-1} & y_{n}
\end{array}\right) .
$$

For any representation $\varrho$ of $\mathfrak{g}$, the spectral curve $S_{\varrho}$ defined by the equation $\operatorname{det} \varrho(A(z)-\lambda I)=0$ is independent of time (i.e., is a conserved quantity of the system). The spectral curve is a finite cover of $\mathbb{P}^{1}$ which for generic $z$ parameterizes the 
eigenvalues of $\varrho(A(z))$. While the eigenvalues are conserved by the system, the eigenvectors are not. The eigenvectors of $\varrho(A)$ determine a line bundle on the spectral cover, so an element of $\operatorname{Jac}\left(S_{\varrho}\right)$. The flow of the system is linearized on this Jacobean. Since the original system of equations did not depend on a choice of representation $\varrho$, the flow is actually linearized on an Abelian variety which is a subvariety of $\operatorname{Jac}\left(S_{\varrho}\right)$ for every $\varrho$.

In fact, instead of considering each spectral cover we can look at the cameral cover $X \rightarrow \mathbb{P}^{1}$. This is constructed as a pullback to $\mathbb{P}^{1}$ of the cover $t \rightarrow t / G$, where $G$ is the Weyl group of $\mathfrak{g}$. This cover is pulled back by the rational map $\mathbb{P}^{1} \rightarrow \mathfrak{t} / G$ defined by the class of $A(z)$ under the adjoint action of the corresponding Lie group. (For $A(z)$ a regular semisimple element of $\mathfrak{s}(n)$, this map sends $z$ to the unordered set of eigenvalues of $A(z)$.) Thus, the cameral cover is a finite Galois cover of $\mathbb{P}^{1}$ whose Galois group $G$ is the Weyl group of $\mathfrak{g}$. The flow of the Toda system is linearized on the Prym of this cover corresponding to the representation of $G$ on $t^{*}$. This is an $r$-dimensional representation, where $r$ is the rank, so the dimension of this Prym is

$$
r(-1)+\sum_{k=1}^{N}\left(r-\left(\operatorname{dim} \mathrm{t}^{H_{k}}\right)\right) \frac{R_{H_{k}}}{2} .
$$

The ramification of this cover has been analyzed in $[6,11]$. There are $2 r$ branch points where the inertial group $H$ is $\mathbb{Z} / 2 \mathbb{Z}$ generated by one reflection, so for each of these $\operatorname{dim}^{H}$ is $r-1$. There are also two points $(z=0$ and $\infty)$ where the inertial group $H$ is generated by the Coxeter element, the product of the reflections corresponding to the simple roots. This element of $G$ does not fix any element of $t$, so for these two points $\operatorname{dim} t^{H}=0$. Thus the dimension of the Prym is

$$
-r+(r-(r-1)) \frac{2 r}{2}+(r-0) \frac{2}{2}=r
$$

Since the original system of equations had a $2 r$-dimensional phase space, this is the answer that we want.

3.2. Hitchin systems. Hitchin [9] showed that the cotangent bundle to the moduli space of semistable vector bundles on a curve $Y$ has the structure of an algebraically completely integrable system. His proof, later extended to principal $\mathscr{G}$ bundles with any reductive Lie group $\mathscr{G}[7,13]$, uses the fact that this moduli space is equivalent (by deformation theory) to the space of Higgs pairs, pairs $(P, \phi)$ of a principal bundle, and an endomorphism $\phi \in H^{0}\left(Y, \operatorname{ad}(P) \otimes \omega_{Y}\right)$. As in the case of the Toda system, the key construction is of a cameral cover of $Y$. The eigenvalues of $\phi$, which are sections of the line bundle $\omega_{Y}$, determine a spectral cover of $Y$ in the total space of the bundle. The eigenvectors determine a line bundle on this spectral cover. The Hitchin map sends a Higgs pair $(P, \phi)$ to the set of coefficients of the characteristic polynomial. Each coefficient is a section of a power of $\omega_{Y}$, so the image of the Hitchin map is $B:=\bigoplus_{i=1}^{r} H^{0}\left(Y, \omega_{Y}^{\otimes d_{i}}\right)$, where the $d_{i}$ are the degrees of the basic invariant polynomials of the Lie algebra $\mathfrak{g}$.

Again, we can consider instead the cameral cover $X_{b} \rightarrow Y$, which is obtained as a pullback to $Y$ via $\phi$ of $\mathfrak{t} \otimes \omega_{Y} \rightarrow \mathfrak{t} \otimes \omega_{Y} / G$. The generic fiber of the Hitchin map is 
isogenous to $\operatorname{Prym}_{\mathfrak{t}}(X)$, which has dimension

$$
r(g-1)+\sum_{k=1}^{N}\left(r-\left(\operatorname{dim} t^{H_{k}}\right)\right) \frac{R_{H_{k}}}{2} .
$$

By looking at the generic fiber, we can restrict our attention to cameral covers where the only ramification is of order two, with inertial group $H$ generated by one reflection. The last piece of information we need to compute the dimension is the degree of the branch divisor of $X \rightarrow Y$.

The cover $t \otimes \omega_{Y} \rightarrow t \otimes \omega_{Y} / G$ is ramified where any of the roots, or their product, is equal to zero. There are $(\operatorname{dim} \mathscr{G}-r)$ roots, so this defines a hypersurface of degree $(\operatorname{dim} \varphi-r)$ in the total space of $\omega_{Y}$. The ramification divisor of $X \rightarrow Y$ is the intersection of this hypersurface with the section $\phi$, which is the divisor corresponding to the line bundle $\omega_{Y}^{\otimes\left(\operatorname{dim}^{\mathscr{G}}-r\right)}$. Thus the degree of the branch divisor will be $(\operatorname{dim} \varphi-r)(2 g-2)$.

Combining all of this information, we see that the dimension of the Prym is

$$
\begin{aligned}
\operatorname{dim}_{\operatorname{Prym}_{\mathfrak{t}}(X)} & =r(g-1)+(r-(r-1)) \frac{(\operatorname{dim} \mathscr{G}-r)(2 g-2)}{2} \\
& =r(g-1)+(\operatorname{dim} \mathscr{G}-r)(g-1) \\
& =\operatorname{dim} \mathscr{G}(g-1) .
\end{aligned}
$$

By comparison, the dimension of the base space is

$$
\sum_{i=1}^{r} h^{0}\left(Y, \omega_{Y}^{d_{i}}\right)
$$

The sum of the degrees $d_{i}$ of the basic invariant polynomials of $\mathfrak{g}$ is the dimension of a Borel subalgebra, $(\operatorname{dim} \varphi+r) / 2$. For $g>1$, Riemann-Roch gives

$$
\sum_{i=1}^{r} h^{0}\left(Y, \omega_{Y}^{d_{i}}\right)=\sum_{i=1}^{r}\left(2 d_{i}-1\right)(g-1)=(\operatorname{dim} \mathscr{G}+r-r)(g-1)=\operatorname{dim} \mathscr{G}(g-1) .
$$

Which, as Hitchin said, "somewhat miraculously" turns out to be the same thing.

Markman [10] and Bottacin [4] generalized the Hitchin system by twisting the line bundle $\omega_{Y}$ by an effective divisor $D$. The effect of this is to create a family of integrable systems, parameterized by the residue of the Higgs field $\phi$ at $D$. The base space of each system is a fiber of the map

$$
\begin{gathered}
B:=\bigoplus_{i=1}^{r} H^{0}\left(Y, \omega_{Y}(D)^{\otimes d_{i}}\right) \\
\downarrow \bar{B}:=\text { the space of possible residues at } D,
\end{gathered}
$$

which sends the set of $r$ sections in $B$ to its set of residues at $D$. At each point of $D$, there are $r$ independent coefficients, so the dimension of $\bar{B}$ is $r(\operatorname{deg} D)$. Thus the base 
space of each system has dimension

$$
\begin{aligned}
\operatorname{dim} B-\operatorname{dim} \bar{B} & =\sum_{i=1}^{r} h^{0}\left(Y, \omega_{Y}(D)^{\otimes d_{i}}\right)-r(\operatorname{deg} D) \\
& =\sum_{i=1}^{r}\left(d_{i}(2 g-2+\operatorname{deg} D)-(g-1)\right)-r(\operatorname{deg} D) \\
& =\frac{1}{2}(\operatorname{dim} \varphi+r)(2 g-2+\operatorname{deg} D)-r(g-1)-r(\operatorname{deg} D) \\
& =(\operatorname{dim} \mathscr{G})(g-1)+\frac{\operatorname{dim} \varphi-r}{2} \operatorname{deg} D .
\end{aligned}
$$

Markman [10] showed that the generic fiber of this system is again isogenous to $\operatorname{Prym}_{\mathfrak{t}}(X)$, where $X$ is a cameral cover of the base curve $Y$. The construction of the cameral cover is similar to the case of the Hitchin system, except that $\phi$ is a section of $\operatorname{ad}(P) \otimes \omega_{Y}(D)$. Thus the ramification divisor is $\left(\omega_{Y}(D)\right)^{\otimes\left(\operatorname{dim} \varphi^{-}-r\right)}$, and the dimension is

$$
\begin{aligned}
\operatorname{dim} \operatorname{Prym}_{\mathfrak{t}}(X) & =r(g-1)+\frac{(\operatorname{dim} \mathscr{G}-r)(2 g-2+\operatorname{deg} D)}{2} \\
& =\operatorname{dim} \mathscr{G}(g-1)+\frac{(\operatorname{dim} \mathscr{G}-r)}{2} \operatorname{deg} D .
\end{aligned}
$$

Again, this is the same dimension as the base of the system.

ACKNOWLEDGEMENTS. This work appeared as part of a Ph.D. thesis at the University of Pennsylvania. The author would like to thank her thesis advisor, Ron Donagi, for suggesting this project and for many helpful discussions. Thanks are also due to David Harbater, Eyal Markman, and Leon Takhtajan.

\section{REFERENCES}

[1] M. Adler and P. van Moerbeke, Completely integrable systems, Euclidean Lie algebras, and curves, Adv. in Math. 38 (1980), no. 3, 267-317. MR 83m:58041. Zbl 455.58017.

[2] P. S. Aspinwall, Aspects of the hypermultiplet moduli space in string duality, J. High Energy Phys. (1998), no. 4, Paper 19, 27 pp. (electronic). MR 99e:81170. Zbl 958.81070.

[3] Y. G. Berkovich and E. M. Zhmud', Characters of Finite Groups. Part 1, translations of Mathematical Monographs, vol. 172, American Mathematical Society, Rhode Island, 1998. MR 98m:20011. Zbl 934.20008.

[4] F. Bottacin, Symplectic geometry on moduli spaces of stable pairs, Ann. Sci. Ecole Norm. Sup. (4) 28 (1995), no. 4, 391-433. MR 96h:14008. Zbl 864.14004.

[5] R. Donagi, Decomposition of spectral covers, Asterisque (1993), no. 218, 145-175, Journees de geometrie algebrique d'Orsay, France, juillet 20-26. MR 95f:14065. Zbl 820.14031.

[6] R. Y. Donagi, Seiberg-Witten integrable systems, Algebraic Geometry-Santa Cruz 1995 (Rhode Island), Proc. Sympos. Pure Math. Part 2, vol. 62, Amer. Math. Soc., 1997, pp. 3-43. MR 99c:58066. Zbl 896.58057.

[7] G. Faltings, Stable G-bundles and projective connections, J. Algebraic Geom. 2 (1993), no. 3, 507-568. MR 94i:14015. Zbl 790.14019.

[8] W. Fulton and J. Harris, Representation Theory: a First Course, Graduate Texts in Mathematics, Springer-Verlag, New York, 1991, Readings in Mathematics. MR 93a:20069. Zbl 744.22001. 
[9] N. J. Hitchin, Stable bundles and integrable systems, Duke Math. J. 54 (1987), no. 1, 91114. MR 88i:58068. Zbl 627.14024.

[10] E. Markman, Spectral curves and integrable systems, Compositio Math. 93 (1994), no. 3, 255-290. MR 95k:14013. Zbl 824.14013.

[11] A. McDaniel and L. Smolinsky, A Lie-theoretic Galois theory for the spectral curves of an integrable system. II, Trans. Amer. Math. Soc. 349 (1997), no. 2, 713-746. MR 97j:58065. Zbl 868.58046.

[12] J.-Y. Merindol, Varietes de Prym d'un revetement galoisien [Prym varieties of a Galois covering], J. Reine Angew. Math. 461 (1995), 49-61 (French). MR 96b:14035. Zbl 814.14043.

[13] R. Scognamillo, An elementary approach to the abelianization of the Hitchin system for arbitrary reductive groups, Compositio Math. 110 (1998), no. 1, 17-37. MR 99b:14013. Zbl 915.14007.

Amy E. Ksir: MAthematics Department, State University of New York at Stony Brook, STONY BROOK, NY 11794, USA

E-mail address: ksir@math.sunysb.edu 


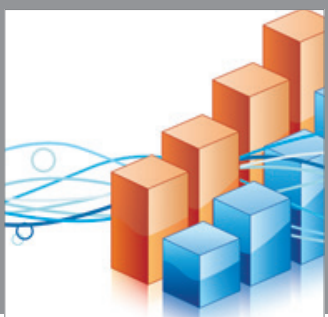

Advances in

Operations Research

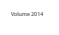

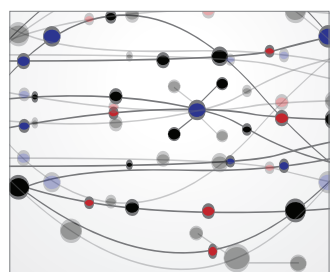

\section{The Scientific} World Journal
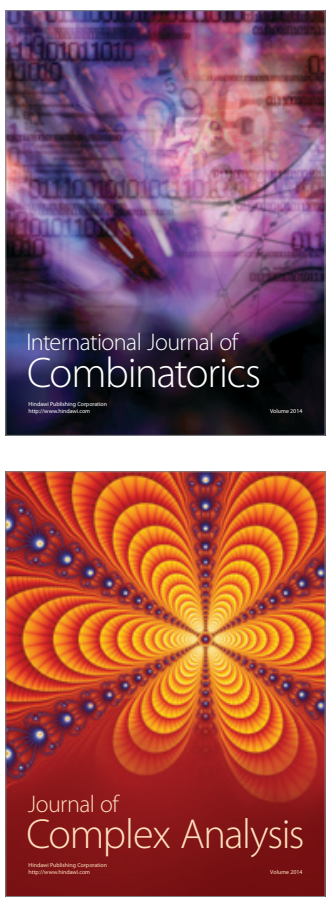

International Journal of

Mathematics and

Mathematical

Sciences
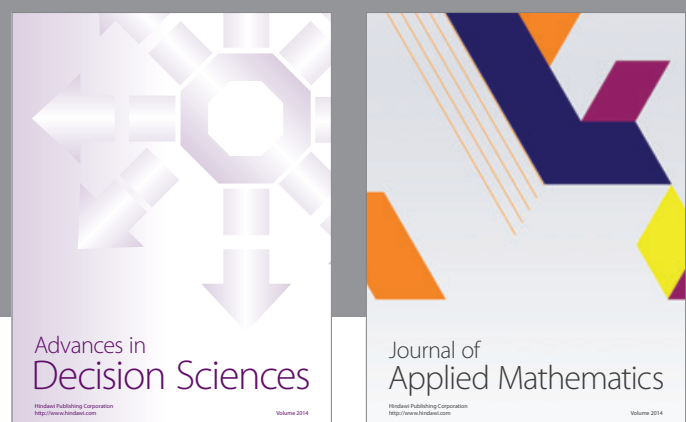

Journal of

Applied Mathematics
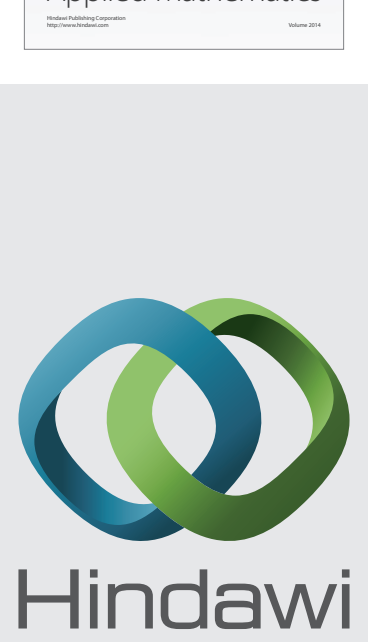

Submit your manuscripts at http://www.hindawi.com
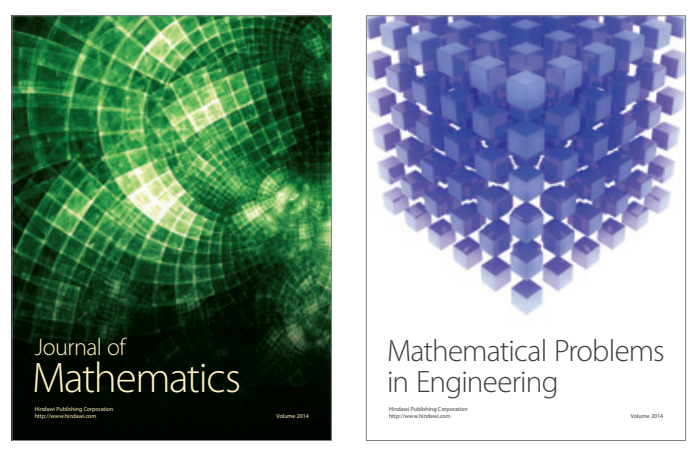

Mathematical Problems in Engineering
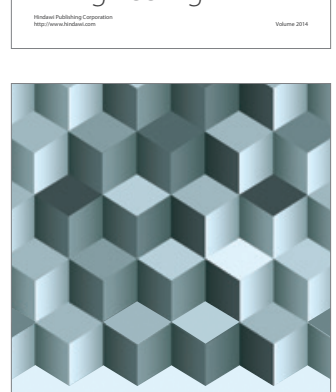

Journal of

Function Spaces
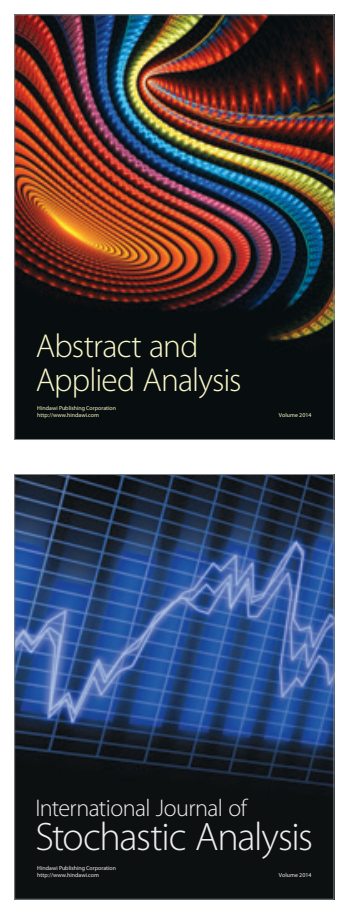

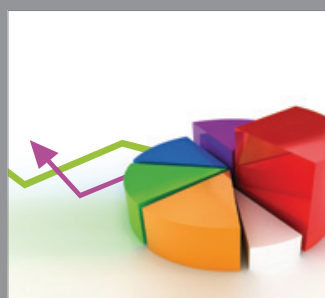

ournal of

Probability and Statistics

Promensencen
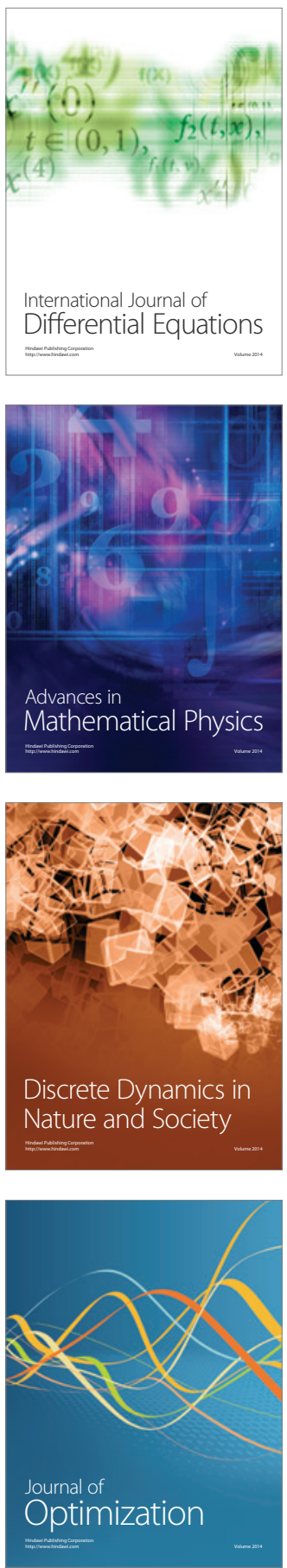